Editorial

\title{
Special Issue Editorial: Ionic Liquid Crystals
}

\author{
Giacomo Saielli (i) \\ CNR Institute on Membrane Technology, Unit of Padova, and Department of Chemical Sciences, University of \\ Padova, via Marzolo, 1 - 35131 Padova, Italy; giacomo.saielli@unipd.it
}

Received: 23 May 2019; Accepted: 26 May 2019; Published: 27 May 2019

The term "Ionic Liquid Crystals" (ILCs) clearly results from the blending of the well-known "Ionic Liquids" (ILs) and "Liquid Crystals" (LCs) names of the corresponding materials. The concatenating word Liquid is crucial since this is the property that makes all three types of materials so important: Without the key feature of being fluid, there would be not such notable interest in the phase behavior of either an ionic or molecular solid. Coincidentally, both ILs and LCs were discovered in 1888 [1,2] and they remained just an academic curiosity for many decades, until industrial applications eventually took off. This happened during the 1970s for liquid crystals, after the synthesis of a new family of LCs based on cyanobiphenyls, stable to oxidation and light irradiation [3]; similarly, ILs had to wait until the discovery of air and water stable imidazolium salts in the 1990s [4] before they started to become appealing for industrial processes.

Thermotropic Ionic Liquid Crystals were first officially reported some 50 years later [5], compared to the "parent" compounds, but in fact they have been known since ancient times, since soaps, that is metal alkanoates, exhibit ionic liquid crystal phases. ILCs can be viewed as ILs that, at some intermediate temperature between the isotropic liquid and the crystal phase, also exhibit a liquid-crystalline (LC) mesophase. Most of the ILC compounds known today are composed by the same type of cations and anions usually found in ILs; however, because of the presence of relatively long carbon chains, micro-segregation leads to the formation of LC phases, almost invariably of smectic type, that is layered. Other LC phases encountered in ionic systems are columnar and cubic ones. In contrast, the ionic nematic phase is extremely rare and the quest for a family of compounds showing a stable ionic nematic phase, near room temperature and with a relatively large thermal range of stability, is an active field of research. In any case, even for the most common kinds of ILCs, the detailed understanding of the relationship between the molecular structure of cation and anion and the phase behavior and thermal stability is far from being understood.

The potential applications of ILCs span a wide range of options and they have been tested in several proof-of-principle devices. The special solvation properties of ILs, combined with the partial orientational and/or translational order of LCs, make ILCs promising media in all cases where a transport of mass and/or charge is needed. There is a main drawback, though, that is the relatively high viscosity. This is the reason why deeper and more thorough investigations of ILCs are necessary, in order to understand how the many details of the molecular structure of cations and anions affect the macroscopic properties and the thermal range and type of mesophases of ILCs. These, in fact, depend on the interplay of a number of steric, van der Waals, H-bonding, and electrostatic interactions and their modeling is an arduous task.

This Special Issue on Ionic Liquid Crystals aims at gathering together some of the specialists working with ILCs, to shed light on the properties and behavior of ILCs. The papers cover many aspects of ILCs science and technology from organic to computational chemistry, from physical chemistry to engineering applications, thus reflecting the many interests of the community of scientists active in the field.

In Reference [6], Liu et al. have investigated the ability of nanoparticles to trap ionic impurities in a LC cell and therefore the possibility to reduce the residual DC current. The residual direct current 
voltage caused by the accumulation of mobile ions is here prohibited. Although the system investigated is not stricto sensu a thermotropic ILC, the work clearly highlights the importance of the precise control and understanding of ionic interaction in LC mesophase for real applications.

In Reference [7], Bhowmik and co-workers report the synthesis and characterization of a series of viologen-based ionic liquid crystals having $4-n$-alkylbenzenesulfonates as counter-anions. Viologens have interesting redox and electrochromic properties, therefore the investigation of their mesophase behavior is of utmost importance in view of possible applications.

In Reference [8], Laschat, Giesselmann and co-workers report the synthesis and characterization of discotic ILCs based on crown ethers. The systems formed columnar mesophases and the authors observed an improved electronic transport, namely the hole mobility, in macroscopically aligned thin films. They excluded the presence of channels for fast cation transport; rather they found that the ion migration is dominated by non-coordinating anions propagating trough the ordered medium.

An interesting investigation of the relationship between molecular structure of the constituent cations and anions and the phase behavior of the material, is reported by Goossens et al. in Reference [9]. They prepared a series of 4,5-bis(n-alkyl)azolium salts and studied their behavior. The authors observed that the presence of substituents on the 4- and 5-positions of the imidazolium ring increases the melting points and lowers the clearing points compared to the 1,3-disubtituted analogues.

An entirely different perspective on ILCs is presented by Cao and Wang. In their paper, Reference [10], they investigated imidazolium salts using fully atomistic molecular dynamics (MD) simulations. They prepared different crystal structures of 1-tetradecyl-3-methylimidazolium nitrate and heated them up to the transition into the smectic phase. They observed that all systems melt into the same SmA phase. The systems go through a metastable state which is characterized by an orientation of the chains almost perpendicular to the smectic layers. The power of MD simulations is therefore highlighted by the possibility to study phases not accessible by experiments and to rationalize their stability.

Nuclear Magnetic Resonance spectroscopy is a fundamental experimental technique to investigate ordered phases; measuring ${ }^{13} \mathrm{C}-{ }^{1} \mathrm{H}$ dipolar couplings of samples in an orientationally ordered medium allows to obtain orientational order parameters of the corresponding $\mathrm{C}-\mathrm{H}$ bonds. Based on this technique, Dvinskikh and co-worker in Reference [11] have analyzed the orientational order of the thermotropic ILC 1-tetradecyl-3-methylimidazolium nitrate in the thermal range of stability of the smectic phase. They reported a significantly lower value of the orientational order parameters compared to conventional non-ionic LC phases.

Finally, we also presented a contribution concerned with MD simulations, using highly coarse-grained models, of ILCs [12]. We considered a mixture of ellipsoidal particles based on the Gay-Berne potential, positively charged to represent the cations, and spherical Lennard-Jones particles negatively charged to represent the anions. Though extremely simplified, the investigation of the phase diagram of such a model system showed the appearance of a very stable ionic nematic phase between the isotropic phase and the smectic phase.

To conclude, I believe that this Special Issue on Ionic Liquid Crystals touches on the latest advancements in several aspects related to ILCs science: Synthesis of novel compounds, spectroscopic studies, MD simulations, and investigations of both structural and dynamic properties. I wish to express my deepest and sincere gratitude to all authors who contributed, for having submitted manuscripts of such excellent quality. I also wish to thank the Editorial Office of Crystals for the fast and professional handling of the manuscripts during the whole submission process and for the help provided.

Conflicts of Interest: The authors declare no conflict of interest. 


\section{References}

1. Gabriel, S.; Weiner, J. Ueber einige Abkömmlinge des Propylamins. Ber. Dtsch. Chem. Ges. 1888, 21, 2669-2679. [CrossRef]

2. Reinitzer, F. Beiträge zur Kenntniss des Cholesterins. Monatsh. für Chem. (Wien) 1888, 9, 421-441. [CrossRef]

3. Gray, G.W.; Harrison, K.J.; Nash, J.A. New family of nematic liquid crystals for displays. Electr. Lett. 1973, 9 , 130-131. [CrossRef]

4. Wilkes, J.S.; Zaworotko, M.J. Air and Water Stable 1-Ethyl-3-methylimidazolium Based Ionic Liquids. J. Chem. Soc. Chemm. Commun. 1992, 965-967. [CrossRef]

5. Knight, G.A.; Shaw, B.D. Long-Chain Alkylpyridines and Their Derivatives. New Examples of Liquid Crystals. J. Chem. Soc. 1938, 682-683. [CrossRef]

6. Liu, Y.; Sang, J.; Liu, H.; Xu, H.; Zhao, S.; Sun, J.; Lee, J.H.; Jeong, H.-C.; Seo, D.-S. Decreasing the Residual DC Voltage by Neutralizing the Charged Mobile Ions in Liquid Crystals. Crystals 2019, 9, 181. [CrossRef]

7. Bhowmik, P.K.; Chang, A.; Kim, J.; Dizon, E.J.; Principe, R.C.G.; Han, H. Thermotropic Liquid-Crystalline Properties of Viologens Containing 4-n-alkylbenzenesulfonates. Crystals 2019, 9, 77. [CrossRef]

8. Staffeld, P.; Kaller, M.; Ehni, P.; Ebert, M.; Laschat, S.; Giesselmann, F. Improved Electronic Transport in Ion Complexes of Crown Ether Based Columnar Liquid Crystals. Crystals 2019, 9, 74. [CrossRef]

9. Goossens, K.; Rakers, L.; Shin, T.J.; Honeker, R.; Bielawski, C.W.; Glorius, F. Substituted Azolium Disposition: Examining the Effects of Alkyl Placement on Thermal Properties. Crystals 2019, 9, 34. [CrossRef]

10. Cao, W.; Wang, Y. Phase Behaviors of Ionic Liquids Heating from Different Crystal Polymorphs toward the Same Smectic-A Ionic Liquid Crystal by Molecular Dynamics Simulation. Crystals 2019, 9, 26. [CrossRef]

11. Dai, J.; Kharkov, B.B.; Dvinskikh, S.V. Molecular and Segmental Orientational Order in a Smectic Mesophase of a Thermotropic Ionic Liquid Crystal. Crystals 2019, 9, 18. [CrossRef]

12. Margola, T.; Satoh, K.; Saielli, G. Comparison of the Mesomorphic Behaviour of 1:1 and 1:2 Mixtures of Charged Gay-Berne GB(4.4,20.0,1,1) and Lennard-Jones Particles. Crystals 2018, 8, 371. [CrossRef]

(C) 2019 by the author. Licensee MDPI, Basel, Switzerland. This article is an open access article distributed under the terms and conditions of the Creative Commons Attribution (CC BY) license (http://creativecommons.org/licenses/by/4.0/). 